\title{
Is Automated Weaning Superior to Manual Spontaneous Breathing Trials?
}

\author{
Steven R Holets RRT and John J Marini MD
}

\author{
Introduction \\ Evidence in Support of Automated Weaning Modes: Pro \\ Automated Weaning Will Improve Best Practice \\ Automated Weaning Will Reduce Work Load \\ Is Automated Weaning Superior to Manual Spontaneous Trials? No \\ Conclusions
}

\begin{abstract}
Weaning from mechanical ventilation involves the reduction or withdrawal of ventilatory support in proportion to the patient's ability to sustain spontaneous ventilation. Protocolized weaning has been shown to reduce weaning duration; however, its weakness lies in the reliance on human intervention. Automated weaning is theoretically superior to manual weaning because of its ability to rapidly recognize deviations from desired behavior and enforce compliance with a standardized weaning strategy unencumbered by external influences. Whether currently available methods for automated weaning fulfill that potential to achieve superiority depends on patient type, care environment, and cause of ventilator dependence. Key words: automated weaning; spontaneous breathing trial; automated modes; weaning; mechanical ventilation. [Respir Care 2016;61(6):749-760. (C) 2016 Daedalus Enterprises]
\end{abstract}

\section{Introduction}

Automated weaning is theoretically superior to manual weaning because of its ability to rapidly recognize deviations from targeted parameters and therefore enforce compliance with a standardized weaning strategy unencumbered by external influences. Whether currently available

Mr Holets is affiliated with the Department of Respiratory Care, Mayo Clinic, Rochester, Minnesota. Dr Marini is affiliated with the Department of Pulmonary/Critical Care, Regions Hospital, St Paul, Minnesota.

Mr Holets discloses a relationship with Resmed. Dr Marini has no conflicts to disclose.

Mr Holets and Dr Marini presented a version of this paper at the 54th RESPIRATORY CARE Journal Conference, "Respiratory Care Controversies III," held June 5-6, 2015, in St Petersburg, Florida.

Correspondence: Steven R Holets RRT, Mayo Clinic, 200 2nd Ave SW, Rochester, MN 55905. E-mail: holets.steven@mayo.edu.

DOI: $10.4187 /$ respcare.04329 methods for automated weaning fulfill that potential to achieve superiority may depend, however, on patient type, care environment, and cause of ventilator dependence. What follows is a discussion of the arguments and evidence on both sides of the question.

\section{Evidence in Support of Automated Weaning Modes: Pro}

Automated weaning modes first appeared on ventilators more than 2 decades ago. However, few scientific studies supporting manufacturers' claims of improved outcomes accompanied their appearance. An early examination of the evidence at the time conceded that in fact "there is none." 1 Lack of randomized controlled trials, misunderstanding of mode functionality, and the sometimes overzealous marketing left many reluctant to incorporate potentially beneficial technology and, even worse, encouraged caregivers to continue to employ inferior weaning modalities. $^{2}$ This led clinicians and leaders in respiratory care to call for clear scientific evidence of improved outcomes 
Table 1. Comparative Weaning Trials Using Adaptive Support Ventilation and SmartCare/PS

\begin{tabular}{|c|c|c|c|c|c|}
\hline First Author & $\begin{array}{l}\text { Automated } \\
\text { Mode }\end{array}$ & Population & Comparator & $\begin{array}{l}\text { Main } \\
\text { Outcome }\end{array}$ & Findings \\
\hline Kirakli $^{5}$ & ASV & $\begin{array}{l}97 \text { subjects with } \\
\text { COPD }\end{array}$ & $\begin{array}{l}\text { Protocol: PSV with } \\
\text { 2-h SBT }\end{array}$ & $\begin{array}{l}\text { Extubation } \\
\text { success }\end{array}$ & $\begin{array}{l}\text { ASV shortened weaning time: } \\
24 \text { h vs } 73 \mathrm{~h}(P=.041)\end{array}$ \\
\hline Lellouche $^{6}$ & SmartCare/PS & $\begin{array}{l}147 \text { mixed at } 5 \\
\text { European hospitals }\end{array}$ & $\begin{array}{l}\text { Protocol: PSV and } \\
\text { SBT with T-piece or } \\
\text { PSV + PEEP }\end{array}$ & $\begin{array}{l}\text { Weaning } \\
\text { duration }\end{array}$ & $\begin{array}{l}\text { Shorter time to successful } \\
\text { extubation with SmartCare/PS } \\
3 \mathrm{~d} \text { vs } 5 \mathrm{~d}(P=.01)\end{array}$ \\
\hline Jouvet ${ }^{7}$ & SmartCare/PS & 20 pediatric & $\begin{array}{l}\text { Clinician-directed } \\
\text { protocol: PSV }\end{array}$ & $\begin{array}{c}\text { Ventilation } \\
\text { duration }\end{array}$ & $\begin{array}{l}\text { SmartCare/PS shorter } \\
\text { ventilation duration: } \\
5.1 \mathrm{~d} \text { vs } 6.7 \mathrm{~d}(P=.33)\end{array}$ \\
\hline Burns $^{8}$ & SmartCare/PS & $\begin{array}{l}92 \text { mixed from } 9 \\
\text { Canadian ICUs }\end{array}$ & Protocol: PSV & $\begin{array}{l}\text { Time to first } \\
\text { successful SBT }\end{array}$ & $\begin{array}{l}\text { SmartCare/PS shorter } \\
\text { median time to SBT } \\
1 \mathrm{~d} \text { vs } 4 \mathrm{~d}(P<.001)\end{array}$ \\
\hline $\begin{array}{l}\text { PSV }=\text { pressure } \\
\text { SBT }=\text { spontanec } \\
\text { ASV }=\text { adaptive }\end{array}$ & $\begin{array}{l}\text { It ventilation } \\
\text { eathing trial } \\
\text { rt ventilation }\end{array}$ & & & & \\
\hline
\end{tabular}

before the acceptance any new mode and to reevaluate the continued use of traditionally unproven modes, such as synchronized intermittent mandatory ventilation. ${ }^{3}$ In response, both for academic and market-driven reasons, a considerable amount of research regarding the efficacy of automated weaning modes has since been conducted, reviewed, and evaluated by recognized experts in the field, professional medical organizations, and government regulatory bodies. Their findings have shed light upon the benefits and potential pitfalls of automated modes and offer recommendations as to how they may be best utilized to improve patient care.

Although an in-depth review of the totality of evidence is beyond the scope of this discussion, several recent publications warrant review. Branson ${ }^{4}$ performed a comprehensive appraisal of closed-loop modes, including the evidence for and against their use to facilitate weaning, in 2012. He found insufficient evidence to support the use of several modes for weaning, although improved oxygenation, airway pressures, number of ventilator manipulations, and identification of weaning readiness favored automated modalities compared with traditional modes. The majority of comparative clinical trials have been conducted using Adaptive Support Ventilation (Hamilton Medical) and SmartCare/PS (Table 1). In adult subjects with COPD, ASV reduced weaning time from 3 to $1 \mathrm{~d}$, whereas in a mixed population, SmartCare/PS (Dräger Medical) shortened weaning duration from 5 to $3 \mathrm{~d}$. Similar improvements were seen in the pediatric population, with reductions from 6.7 to $5.1 \mathrm{~d}$. Other studies had mixed results without improvement in weaning time. Nonetheless, no differences in sedation use, ventilator manipulations, or adverse events were recorded, suggesting that at the very least, automated weaning poses no greater risk than and is equivalent to conventional weaning.
The Canadian Wean Earlier and Automatically with New technology trial (the WEAN study) is the largest multicenter, randomized trial conducted in North America to date. ${ }^{8}$ It compared a standardized weaning protocol with automated weaning in a multidisciplinary population and evaluated clinician, respiratory therapist (RT), and nursing compliance with and perceptions of automated weaning. Median time to the first successful breathing trial, extubation, and successful extubation were all significantly shorter (1-3 h, $P<.01-.02)$ in those receiving automated weaning, with fewer tracheostomies and episodes of extended ventilation. Furthermore, less use of sedation and analgesia and fewer days with extreme sedation scoring suggest that automated weaning may facilitate sedation management. Clinician and RT protocol acceptance scores were lower in the automated weaning group, attributed to the reluctance to relinquish ventilatory control and resistance to the implementation of a new technology.

Finally, a large systematic review and meta-analysis by Rose et $\mathrm{al}^{9}$ compared conventional and automated weaning in children and adults. The Cochrane Collaboration quality standards were applied to randomized controlled trials from electronic databases, conference proceedings, and original articles, and 16 studies utilizing protocolized weaning as a comparator were identified. Overall, SmartCare/PS reduced weaning by a geometric mean of $30 \%$ in mixed/medical, but not in surgical, ICU populations. There was no difference in weaning duration with any other modes; however, reductions in time to extubation, prolonged intubations, tracheostomies, and ICU stay all favored automated weaning.

As with any promising new technology, there is a need for continued evaluation and development. Limited data exist in the pediatric population, and further research is needed before widespread acceptance. Other populations, 
including neurological, surgical, and ARDS/refractory hypoxemia also deserve additional attention, since many will require prolonged weaning. However, the evidence to date supports the conclusion that automated weaning modalities are safe and may provide important benefits beyond simply speeding the weaning process.

\section{Automated Weaning Will Improve Best Practice}

Evidence-based medicine and quality improvement are the methodologies for translating knowledge into best practice. Evidence-based medicine focuses on "doing the right things" based on available evidence from research, whereas quality improvement focuses on "doing things right" through systems and processes. ${ }^{10}$ Successfully implemented protocols and guidelines that embrace evidencebased medicine and quality improvement concepts have clearly been shown to reduce practice variability, improve outcomes, decrease costs, improve safety, and augment education. ${ }^{11-13}$ Although weaning protocols have been widely endorsed and adopted as standard of care in many ICUs, ${ }^{14-17}$ adherence to guidelines and protocols continues to be a major hurdle even under the scrutiny of research. ${ }^{18}$ This is due to the fact that no matter how explicitly designed or enthusiastically endorsed, the success of any non-automated protocol is largely dependent on human interaction.

Failure to follow a weaning protocol may in part be due to lack of knowledge. Physician or ICU staff may be responsible for a patient being weaned with little or no formal training on the weaning protocol in place. The emergence of telemedicine and the Electronic ICU has resulted in an increase in complex patients managed remotely and at smaller facilities where experience with ventilated patients may be limited. Even experienced personnel require periodic retraining to maintain proficiency. ${ }^{19}$ Process of care and ICU structure recommendations call for the use of standardized protocols by well-trained multidisciplinary teams. ${ }^{20}$ Implementation of computerized protocols for automated weaning will improve best care, at least in part, by reducing errors caused by inexperience and lack of knowledge.

Successful weaning during manual spontaneous breathing trials (SBTs) depends upon the practitioner's ability to recognize physiologic readiness of the patient for trial initiation, time the trial with other interventions such as a sedation holiday, and appropriately recognize, in a timely fashion, when the patient has either passed or failed the SBT. Recognition of readiness can be difficult even for experienced clinicians. A study by Stroetz and Hubmayr ${ }^{21}$ revealed that in subjects who had failed previous weaning attempts, the ability of physicians to predict successful weaning was only $50 \%$, with physician bias toward continuation of unneeded ventilatory support. In contrast, closed-loop algorithms follow very specific rules in which parameters are adjusted based upon individual patient feedback and are comparable with those selected by experienced clinicians. ${ }^{22,23}$ They assist the practitioner in doing "the right thing" with the additional benefit of providing non-distractible, non-fatigable attention to detail beyond human capability.

Well-established objective clinical criteria provide guidance as to when a patient may be ready to undergo a weaning trial. The use of combined sedation and weaning protocols has been shown to shorten the weaning process $^{16,24}$; however, its success is totally dependent upon the coordination of the "sedation holiday" and return of spontaneous breathing with the availability of a practitioner to evaluate and make appropriate changes in a timely fashion. Delays in adjusting ventilator parameters during weaning may cause patient-ventilator asynchrony, reinstitution of sedation, and loss of the window of opportunity for successful extubation..$^{25}$

Real world circumstances routinely prevent even the best-intentioned practitioner from doing "the right thing at the right time." Increased work load, family interactions, emergencies, patient admissions, transports, and other distractions are just a few examples of things that may interfere with the practitioner's bedside presence when needed. The advantage of automated weaning modalities is their ability to provide full support before weaning, recognize spontaneous efforts almost immediately, and continuously assess and adjust settings up or down accordingly based on individualized patient feedback, without vulnerability to outside distractions.

\section{Automated Weaning Will Reduce Work Load}

Staffing shortages and the increasing number of patients requiring mechanical ventilation may be the most compelling argument for automated weaning. It has been demonstrated that work load has a direct effect on patient outcomes. ${ }^{26,27}$ Current respiratory therapist workforce shortages already exist, as highlighted in a 2006 survey of 30 hospitals. ${ }^{28}$ They reported an average staffing level of 10.8 beds/respiratory therapist, exceeding the accepted preferred ratio of 9.4, with a perceived need for an additional 1.3 respiratory therapists in current staffing levels. The ratio of respiratory therapists to ventilated patients was not reported; however, some states have set the maximum ratio at 1:4.29 To meet this requirement, some hospitals have adopted unpopular mandatory overtime policies. Of the 30 hospitals surveyed, 7 utilized mandatory overtime on at least a monthly basis. Since automated weaning modes have been shown to decrease the number of ventilator manipulations, their use should translate into a reduction in work load for already overburdened staff. Future projections of the need for mechanical ventilation estimate an 
annual growth rate of 5\% due mainly to the aging baby boomer population. ${ }^{30}$ Those requiring prolonged mechanical ventilation consume two thirds of total hospital resources devoted to mechanical ventilation. Their numbers are expected to double by 2020 , with projected costs reaching $\$ 60$ billion. ${ }^{30}$ The average additional daily ICU cost of patients requiring mechanical ventilation is $\$ 1,500 .{ }^{31}$ Delayed extubation increases the incidence of complications with increases in hospital costs of $\$ 29,057 /$ incidence. $^{32}$ Although the price of adding automated weaning software may be several thousand dollars per ventilator, a reduction of only 1-2 ventilator days in just a few patients would easily offset the additional expense.

Last, there is the threat of a mass casualty disaster. Significant financial resources and logistical preparation have been expended in planning for various scenarios in which personnel and supply resources will be stretched to their limits. ${ }^{33}$ Although ventilators have been purchased and stockpiled for future mass casualty emergencies, the availability of personnel with sufficient skills to manage them is of concern, ${ }^{33,34}$ reinforcing the vital role for respiratory therapists in disaster planning. One possible solution would be to have "respiratory extenders" oversee basic ventilator operation under the supervision of a therapist, ${ }^{35}$ liberating skilled RT practitioners to provide valuable respiratory services to the more critically ill. Automated weaning modalities would enhance this concept. By having at least a portion of the current ventilator inventory in use equipped with automated modalities, the need for manual manipulations would be reduced, and the potential for errors from inadequately trained personnel would be minimized.

Automated weaning modalities are superior to manual spontaneous trials in their ability to recognize changes in breathing patterns and adjust settings quickly and without human intervention. Their utilization should not be viewed as a relinquishment of control but as a means to improve patient care through the standardization of ventilator management. The growing number of patients requiring mechanical ventilation combined with a diminishing workforce makes the use of automated weaning modalities more a medical and financial necessity than an expensive and unnecessary luxury.

\section{Is Automated Weaning Superior to Manual Spontaneous Trials? No}

An undeniable rationale (if not current justification) exists for "closing the loop" on ventilator support and its withdrawal (weaning). There are worrisome pressures in modern medical practice, as we face the likelihood of increased demand for mechanical ventilation in our aging population and confront staffing issues, ${ }^{19,36,37}$ the mandate to lower costs, ${ }^{38}$ the widespread failure to implement best
Table 2. Important Considerations Before Extubation

\begin{tabular}{l}
\hline \hline Electrolytes \\
Cardiovascular status \\
Nutrition \\
Hydration/Fluid Balance \\
Distension \\
Comfort \\
Infection \\
Secretions \\
Position \\
Anxiolysis \\
Sedation status \\
Sleep
\end{tabular}

practices, and the need for closer patient surveillance so as to intervene in a timely fashion. Well-designed care protocols, if carefully followed by trained health-care professionals, speed the process of liberation from invasive ventilation. ${ }^{39,40}$ One might logically ask why not automate protocolized weaning? The short answer is that, as presently implemented, automation does not incorporate all of the key variables that determine success or failure.

Present day automated algorithms rely primarily on assessing respiratory mechanics, patterns of breathing, and gas exchange, each measure of which has questionable reliability when assessed over brief periods and none of which is currently integrated with unmonitored data coming from the highly influential cardio-hemodynamic axis. The drivers of automated weaning are not fine tuned to recognize trends in response, which may be critical in predicting the patient's trajectory toward the ultimate outcome. Furthermore, current systems work only for wellselected and "tee-ed up" populations. Implicit in the question asked in print by Hess and MacIntyre (“... Why are we still weaning ?"41) is the concern that graded withdrawal of machine support itself may not be necessary or wise. If so, we clearly do not need to automate that process.

There are 5 essential steps to ventilator independence: preparation, evaluation, withdrawal of ventilator support, extraction of the endotracheal tube, and peri-extubation care. Attention to the considerations outlined in Table 2, particularly cardiovascular status, fluid balance, secretion clearance, and appropriate mental status, prepares the patient optimally for disconnection of the machine. ${ }^{42-44}$ Some data convincingly argue that once the patient has optimally been prepared, he or she should be kept fully supported until evaluated for extubation potential under the scrutiny of a trained professional by an abrupt "sink or swim" spontaneous breathing trial in which a T-piece or minimal pressure support is utilized. ${ }^{45,46}$ Depending on performance during the trial, the patient is either left connected and fully supported or extubated expeditiously. Whatever the wisdom of gradual withdrawal of machine support, the 
goal of weaning is not only to discontinue invasive ventilation but also to remove the endotracheal tube. ${ }^{44}$ Evaluation and management of the upper airway both before and after completion of the spontaneous breathing trial comprises the peri-extubation care component of the sequence. No computerized system can assess or manage those crucial pieces of the process. Automated weaning, as currently implemented, only addresses the withdrawal of support from the standpoint of ventilation power and lung performance, with graded interventions gauged primarily by patient breathing pattern response. ${ }^{6,19,47,48}$ Faith is placed implicitly in oxygenation efficiency and the tidal volume and frequency amplitudes to signal reserve or intolerance, whatever the cause of trouble might be: ventilation power, hemodynamics, or oxygenation. ${ }^{48}$ But the inconvenient truth is that the respiratory pattern has never been shown to reliably index hemodynamic or oxygenation distress. Newer systems have begun to integrate gas exchange data, which clearly is a conceptual step forward. Unfortunately, to this point, evidence is very limited regarding their incremental value and performance merits. ${ }^{9}$

It is obvious that automated weaning does little for the preparation phase or for the peri-extubation phases of the weaning process. The causes of postextubation distress include retention of airway secretions, upper airway dysfunction, positive fluid imbalance, and inadequate cardiovascular reserve: congestive failure, arrhythmia, or coronary ischemia. ${ }^{42-44}$ Respiratory distress and central vascular congestion can be precipitated by spontaneous ventilatory effort. ${ }^{49}$ These problems of secretion retention and cardiac dysfunction are often best revealed considerably after extubation has been accomplished, cough effectiveness is tested, and net intrathoracic pressures fall. ${ }^{50}$ Moreover, because separation of the patient from the ventilator may fail because of neuro/psychiatric and sedation issues as well as for respiratory or cardiac reasons, the patient should ideally be evaluated for alertness, command response, and cough effectiveness before acting on any "go ahead" prompt from the automated system, which cannot assess these important characteristics.

On the respiratory side, imbalance of capability with demand may be provoked by postextubation secretion retention, dynamic hyperinflation, bronchospasm, upper airway obstruction, or atelectasis. Each may contribute to gradually developing ventilatory inadequacy and or hypoxemia after extubation. The lungs are challenged on the cardiovascular side as fluid shifts into the central compartment in the transition from positive to negative intrathoracic pressure, and the left ventricle may be excessively afterloaded by strong spontaneous efforts. In fact, worsening lung edema, blood translocation from the periphery to the central compartment, diastolic dysfunction, coronary ischemia, and arrhythmias are frequent causes for the need to re-intubate. ${ }^{43,51}$ Automated weaning before extu- bation might help ease the changeover or avoid abrupt transitions, but comprehensive patient assessment would need to include all variables relevant to postextubation stability, which the attentive bedside caregiver (but not a computer-aided ventilator) can assess and integrate effectively. Although neuropsychiatric distress and sedation issues generally present themselves before attempting extubation, they can arise at any point during the weaning process and may frustrate automation of the process.

Complex mechanisms and critical illnesses often have more than one key element. Perhaps for this reason, the ability of breathing pattern variables alone to predict extubation outcome and monitor "weanability" is weak. ${ }^{51-53}$ In fact, integral components of most automated weaning algorithms, such as the rapid shallow breathing index, although as good or better than any other weanability indicator, do not predict well for the most complicated patients. ${ }^{51,52,54}$ Unmeasured variables, such as the tidal swings and esophageal pressure, outperform the rapid shallow breathing index in head-to-head comparisons. ${ }^{55}$ This advantage is particularly significant when monotonic trends of the esophageal pressure are detected; by comparison, the rapid shallow breathing index tends to plateau to its final value at a very early stage in the failed trial. Moreover, breathing pattern variability, an innate characteristic of health, ${ }^{56}$ is not taken into full account by automated programs. Breath-to-breath variation and complexity of the breathing pattern, key observations not incorporated in today's automated algorithms, have been correlated with ventilation reserve and improved gas exchange. ${ }^{57-59}$ Conversely, lack of variation, unaccounted for in current algorithms, bodes poorly for weanability or even survival. ${ }^{60}$ Variability tends to decline as the work load/capacity ratio increases and is influenced by the mode and level of support. ${ }^{61}$

The existing literature suggests that the mode of mechanical ventilation used in the weaning attempt is less important than previously thought. ${ }^{1,3,16}$ Without question, however, the modes now available for graded withdrawal of ventilation are much more flexible than in prior years. These include neurally adjusted ventilatory assist, ${ }^{61,62}$ proportional assist ventilation, ${ }^{5,63}$ and complex modes that vary the level of pressure assistance and frequency, depending primarily on targeted values for tidal volume and breathing rate 4,6 (Table 3).

Although there has been progress, we still have a long way to go before automated modes can be relied upon to automatically withdraw ventilator support at an appropriate rate. Most of the automated weaning modes now deployed for clinical use impose important restrictions on the type of patient subjected to them. It is assumed for example, that the patient is hemo-dynamically stable, is welloxygenated with limited concentrations of inspired oxygen, has been adjusted to the appropriate level of sedation 
Table 3. Automated Modes: Good Candidates for Automated Weaning?

\section{SmartCare/PS}

Proportional Pressure Support

Adaptive Support Automode

Intellivent-ASV

PAV

NAVA

ASV $=$ adaptive support ventilation

$\mathrm{PAV}=$ proportional assist ventilation

NAVA $=$ neurally adjusted ventilatory assist

Table 4. Is Automated Weaning Superior?

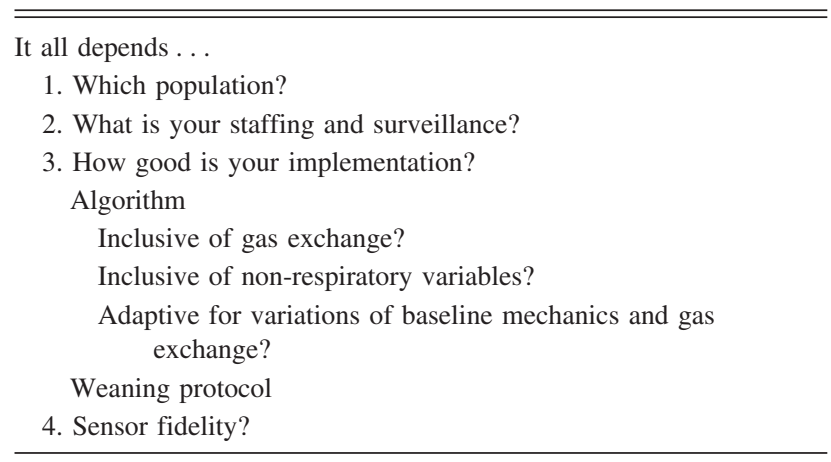

(to "even" the baseline for the automated start), does not have exacerbated illness, does not have unstable respiratory drive or bizarre spontaneous breathing pattern, does not have fever or acid/base disturbance, etc. ${ }^{22,64}$ What's left in the pool of patients to automate are usually not our most troublesome problems, perhaps a reason why evaluations of routine surgical patients do not show a noticeable advantage for weaning automation. ${ }^{64}$

In automated weaning, arbitrarily defined zones of respiratory comfort are identified for tidal volume and frequency. ${ }^{63,65}$ However, many ventilated patients have diseases or chronic conditions that violate these assumptions. ${ }^{66}$ For example, a patient with severe kyphoscoliosis may have a high baseline frequency/tidal volume ratio perfectly compatible with adequate compensation. The same holds true for some patients with chronic neuromuscular disorders. 66

Whether automated weaning proves superior to manual surveillance and adjustment depends on the answers to 4 important questions (Table 4). These include the population in question, the level of staffing and surveillance, the implementation of the automated weaning process to include gas exchange and nonrespiratory (eg, cardiovascular and hemodynamic) variables, and fidelity of the sensor information that drives the adjustments. When the conditions are right and/or the unit is suboptimally staffed, automated weaning appears to hold some advantage over non-automated weaning. ${ }^{8,9,64,67}$ However, when they are not, current systems do not seem to do any better than a well-tuned and well-resourced environment, particularly those that effectively apply protocols. ${ }^{68}$ Patients who do not depend on the machine for reasons of mechanical insufficiency and excessive work load, such as many who undergo surgical care, are not advantaged by automated weaning. Co-management decisions apart from ventilator care, such as those related to levels of sedation, have influenced protocol efficacy in published studies. ${ }^{69,70}$ Indeed, the results of comparative weaning trials depend heavily on how they are conducted. As a classic example, published comparisons of pressure support, synchronized intermittent mandatory ventilation, and T-piece weaning have come to differing conclusions, ${ }^{45,46,71}$ which to some extent can be traced to how each technique was implemented. The same is true in published comparisons of automated methodology with standard conventional methodology.6,47,67 Ideally, funding for comparative trials of any technology would be free of potential conflicts of interest. Unfortunately (but understandably) the majority of the published work favoring commercially available modes of automation has been industry-funded and -supported.

Factors at work that determine the incremental value of automated weaning include the quality of the inputs, the delay until adjustments are implemented, the presence of erratic patterns of ventilation demand, the nature of the patients under study, the skills of practitioners, and the surveillance and protocols available to guide non-automated weaning decisions. There is some concern that automated modes will lead to a lack of valuable interactions that now help to synergize and integrate the efforts of and communications among therapists, nurses, and physicians. Subtle forms of patient-ventilator asynchrony, a factor that influences duration of ventilation, ${ }^{72}$ may not be addressed when the algorithm is not geared specifically to detect them.

Clearly, there are populations where automated weaning (at least as presently configured) would be unsafe, because computer-driven systems do not integrate all of the important variables that can influence patient outcome. As already noted, most studies exclude potentially problematic patients, such as those with neurological impairment and hemodynamic concerns, and little to no data exist for these cohorts. A shortcoming of most automated programs is that there may be failure of appropriate machine response to agitation, secretions, bronchospasm, etc. Automated programs may tend to interrupt expression of an informative trend that otherwise would be detected and promptly addressed by appropriate intervention. As currently implemented, automated algorithms are relatively insensitive to such trends and can neither sense nor integrate the key bedside signs of tolerance or developing problems, such as 
physical appearance, physical examination, electrocardiogram abnormalities, etc.

\section{Conclusions}

There is little question that an attractive potential exists for automated decision support and for perfecting the responsiveness of the ventilator-patient connection. Except in the most sophisticated critical care environments, intelligent automated regulation of ventilatory parameters would be most welcome, since caregivers vary with respect to their skills, diligence, time demands, and resources. Logistical problems are likely to worsen in the near future as the complexity of advanced level care incessantly increases and the availability of caregivers to provide care that is compatible with best practice comes under economic strain. We appear now to be heading in the right direction. However, as currently implemented, automated weaning paradigms leave much to be desired. The populations to which they are applicable are restricted, and their inputs are selective and insufficiently integrated with hemodynamic data and are unable to address important underlying issues that give rise to distress. Moreover, physiologic trajectory (as opposed to snapshot evaluations of present status) is not prioritized, and by design, automated systems do not address important stages of the ventilator liberation process that relate to preparation and extubation. With attention to these limitations, future automated systems may eventually prove of major value as personnel resources are stretched thin. With inherent shortcomings left unaddressed, however, automated weaning cannot be considered a superior methodology for effective and safe care delivery to our most challenging patients, the relatively few who actually need to be weaned at all.

\section{REFERENCES}

1. Branson RD, Johannigman JA. What is the evidence base for the newer ventilation modes? Respir Care 2004;49(7):742-760.

2. Esteban A, Frutos F, Tobin MJ, Alía I, Solsona JF, Valverdú I, et al. A comparison of four methods of weaning patients from mechanical ventilation: Spanish Lung Failure Collaborative Group. N Engl J Med 1995;332(6):345-350.

3. Hess DR. Ventilator modes: where have we come from and where are we going? Chest 2010;137(6):1256-1258.

4. Branson RD. Modes to facilitate ventilator weaning. Respir Care 2012;57(10):1635-1648.

5. Kirakli C, Naz I, Ediboglu O, Tatar D, Budak A, Tellioglu E. A randomized controlled trial comparing the ventilation duration between adaptive support ventilation and pressure assist/control ventilation in medical patients in the ICU. Chest 2015;147(6):1503-1509.

6. Lellouche F, Mancebo J, Jolliet P, Roeseler J, Schortgen F, Dojat M, et al. A multicenter randomized trial of computer-driven protocolized weaning from mechanical ventilation. Am J Respir Crit Care Med 2006;174(8):894-900.

7. Jouvet P, Payen V, Gauvin F, Emeriaud G, Lacroix J. Weaning children from mechanical ventilation with a computer-driven protocol: a pilot trial. Intensive Care Med. 2013;39:(5)919-25.
8. Burns KE, Meade MO, Lessard MR, Hand L, Zhou Q, Keenan SP, Lellouche F. Wean earlier and automatically with new technology (the WEAN study): a multicenter, pilot randomized controlled trial. Am J Respir Crit Care Med 2013;187(11):1203-1211.

9. Rose L, Schultz MJ, Cardwell CR, Jouvet P, McAuley DF, Blackwood B. Automated versus non-automated weaning for reducing the duration of mechanical ventilation for critically ill adults and children: a cochrane systematic review and meta-analysis. Crit Care 2015;19:48.

10. Glasziou P, Ogrinc G, Goodman S. Can evidence-based medicine and clinical quality improvement learn from each other? BMJ Qual Saf 2011;20(Suppl 1):i13-i17.

11. Prasad M, Holmboe ES, Lipner RS, Hess BJ, Christie JD, Bellamy SL, et al. Clinical protocols and trainee knowledge about mechanical ventilation. JAMA 2011;306(9):935-941.

12. Blackwood B, Alderdice F, Burns K, Cardwell C, Lavery G, O'Halloran P. Use of weaning protocols for reducing duration of mechanical ventilation in critically ill adult patients: Cochrane systematic review and meta-analysis. BMJ 2011;342:c7237.

13. Kollef MH, Micek ST. Using protocols to improve patient outcomes in the intensive care unit: focus on mechanical ventilation and sepsis. Semin Respir Crit Care Med 2010;31(1):19-30.

14. Prasad M, Christie JD, Bellamy SL, Rubenfeld GD, Kahn JM. The availability of clinical protocols in US teaching intensive care units. J Crit Care 2010;25(4):610-619.

15. Boles JM, Bion J, Connors A, Herridge M, Marsh B, Melot C, et al. Weaning from mechanical ventilation. Eur Respir J 2007;29(5):10331056.

16. MacIntyre NR, Cook DJ, Ely EW Jr, Epstein SK, Fink JB, Heffner $\mathrm{JE}$, et al. Evidence-based guidelines for weaning and discontinuing ventilatory support: a collective task force facilitated by the American College of Chest Physicians; the American Association for Respiratory Care; and the American College of Critical Care Medicine. Chest 2001;120(6 Suppl):375S-395S.

17. Metcalf AY, Stoller JK, Fry TD, Habermann M. Patterns and factors associated with respiratory care protocol use. Respir Care 2015; 60(5):636-643.

18. Randolph AG, Lacroix J. Randomized clinical trials in pediatric critical care: rarely done but desperately needed. Pediatr Crit Care Med 2002;3(2):102-106.

19. Sinuff T, Cook D, Giacomini M, Heyland D, Dodek P. Facilitating clinician adherence to guidelines in the intensive care unit: a multicenter, qualitative study. Crit Care Med 2007;35(9):2083-2089.

20. Weled BJ, Adzhigirey LA, Hodgman TM, Brilli RJ, Spevetz A, Kline AM, et al. Critical care delivery: the importance of process of care and ICU structure to improved outcomes: an update from the American College of Critical Care Medicine Task Force on Models of Critical Care. Crit Care Med 2015;43(7):1520-1525.

21. Stroetz RW, Hubmayr RD. Tidal volume maintenance during weaning with pressure support. Am J Respir Crit Care Med 1995;152(3): 1034-1040.

22. Dojat M, Harf A, Touchard D, Lemaire F, Brochard L. Clinical evaluation of a computer-controlled pressure support mode. Am J Respir Crit Care Med 2000;161(4 Pt 1):1161-1166.

23. Iotti GA, Polito A, Belliato M, Pasero D, Beduneau G, Wysocki M, et al. Adaptive support ventilation versus conventional ventilation for total ventilatory support in acute respiratory failure. Intensive Care Med 2010;36(8):1371-1379.

24. Girard TD, Kress JP, Fuchs BD, Thomason JW, Schweickert WD, Pun BT, et al. Efficacy and safety of a paired sedation and ventilator weaning protocol for mechanically ventilated patients in intensive care (Awakening and Breathing Controlled trial): a randomised controlled trial. Lancet 2008;371(9607):126-134. 


\section{Automated Weaning vs SBTs}

25. Gilstrap D, MacIntyre N. Patient-ventilator interactions. Implications for clinical management. Am J Respir Crit Care Med 2013; 188(9):1058-1068.

26. Neuraz A, Guérin C, Payet C, Polazzi S, Aubrun F, Dailler F, et al. Patient mortality is associated with staff resources and workload in the ICU: a multicenter observational study. Crit Care Med 2015; 43(8):1587-1594.

27. Gupta P, Giehler K, Walters RW, Meyerink K, Modrykamien AM. The effect of a mechanical ventilation discontinuation protocol in patients with simple and difficult weaning: impact on clinical outcomes. Respir Care 2014;59(2):170-177.

28. Mathews P, Drumheller L, Carlow JJ. Respiratory care manpower issues. Crit Care Med 2006;34(3 Suppl):S32-S45

29. Acute Respiratory Care Service Staffs. California Code of Regulations Title 22, Article 6, Item 70405.

30. Zilberberg MD, de Wit M, Shorr AF. Accuracy of previous estimates for adult prolonged acute mechanical ventilation volume in 2020: update using 2000-2008 data. Crit Care Med 2012;40(1):18-20.

31. Dasta JF, McLaughlin TP, Mody SH, Piech CT. Daily cost of an intensive care unit day: the contribution of mechanical ventilation. Crit Care Med 2005;33(6):1266-1271.

32. Coplin WM, Pierson DJ, Cooley KD, Newell DW, Rubenfeld GD. Implications of extubation delay in brain-injured patients meeting standard weaning criteria. Am J Respir Crit Care Med 2000;161(5): 1530-1536.

33. Rubinson L, Nuzzo JB, Talmor DS, O'Toole T, Kramer BR, Inglesby TV. Augmentation of hospital critical care capacity after bioterrorist attacks or epidemics: recommendations of the Working Group on Emergency Mass Critical Care. Crit Care Med 2005;33(10):23932403.

34. Timbie JW, Ringel JS, Fox DS, Pillemer F, Waxman DA, Moore M, et al. Systematic review of strategies to manage and allocate scarce resources during mass casualty events. Ann Emerg Med 2013;61(6): 677-689.e101.

35. Hanley ME, Bogdan GM. Mechanical ventilation in mass casualty scenarios: augmenting staff: project XTREME. Respir Care 2008; 53(2):176-188; discussion 189.

36. Salsberg E, Grover A. Physician workforce shortages: implications and issues for academic health centers and policymakers. Acad Med 2006;81(9):782-787.

37. Angus DC, Shorr AF, White A, Dremisizov TT, Schmitz RJ, Kelley MA, Committee on Manpower for Pulmonary and Critical Care Societies (COMPACCS). Critical care delivery in the United States: distribution of services and compliance with leapfrog recommendations. Crit Care Med 2006;34(4):1016-1024.

38. Wagner DP. Economics of prolonged mechanical ventilation. Am Rev Respir Dis 1989;140(2 Pt 2):S14-S18.

39. Ely EW, Meade MO, Haponik EF, Kollef MH, Cook DJ, Guyatt GH, Stoller JK. Mechanical ventilator weaning protocols driven by nonphysician health-care professionals: evidence-based clinical practice guidelines. Chest 2001;120(6 Suppl):454S-463S.

40. Kollef MH, Shapiro SD, Silver P, St John RE, Prentice D, Sauer S, et al. A randomized, controlled trial of protocol-directed versus physician-directed weaning from mechanical ventilation. Crit Care Med 1997;25(4):567-574.

41. Hess DR, MacIntyre NR. Ventilator discontinuation: why are we still weaning? Am J Respir Crit Care Med 2011;184(4):392-394.

42. Peñuelas O, Frutos-Vivar F, Fernández C, Anzueto A, Epstein SK, Apezteguía $\mathrm{C}$, et al. Characteristics and outcomes of ventilated patients according to time to liberation from mechanical ventilation. Am J Respir Crit Care Med 2011;184(4):430-437.
43. Epstein SK. Etiology of extubation failure and the predictive value of the rapid shallow breathing index. Am J Respir Crit Care Med 1995; 152(2):545-549.

44. Thille AW, Richard JC, Brochard L. The decision to extubate in the intensive care unit. Am J Respir Crit Care Med 2013;187(12):1294-1302.

45. Esteban A, Frutos F, Tobin MJ, Alía I, Solsona JF, Valverdú I, et al A comparison of four methods of weaning patients from mechanical ventilation. Spanish lung failure collaborative group. N Engl J Med 1995;332(6):345-350.

46. Esteban A, Alía I, Gordo F, Fernández R, Solsona JF, Vallverdú I, et al. Extubation outcome after spontaneous breathing trials with t-tube or pressure support ventilation. Am J Respir Crit Care Med 1997; 156(2 Pt 1):459-465.

47. Schädler D, Engel C, Elke G, Pulletz S, Haake N, Frerichs I, et al. Automatic control of pressure support for ventilator weaning in surgical intensive care patients. Am J Respir Crit Care Med 2012; 185(6):637-644.

48. Yang KL, Tobin MJ. A prospective study of indexes predicting the outcome of trials of weaning from mechanical ventilation. $\mathrm{N}$ Engl J Med 1991;324(21):1445-1450.

49. Lemaire F, Teboul JL, Cinotti L, Giotto G, Abrouk F, Steg G, et al. Acute left ventricular dysfunction during unsuccessful weaning from mechanical ventilation. Anesthesiology 1988;69(2):171-179.

50. Lenique F, Habis M, Lofaso F, Dubois-Randé JL, Harf A, Brochard L. Ventilatory and hemodynamic effects of continuous positive airway pressure in left heart failure. Am J Respir Crit Care Med 1997; 155(2):500-505.

51. Frutos-Vivar F, Ferguson ND, Esteban A, Epstein SK, Arabi Y, Apezteguía $\mathrm{C}$, et al. Risk factors for extubation failure in patients following a successful spontaneous breathing trial. Chest 2006;130(6): 1664-1671

52. Meade M, Guyatt G, Cook D, Griffith L, Sinuff T, Kergl C, et al. Predicting success in weaning from mechanical ventilation. Chest 2001;120(6 Suppl):400S-424S

53. Conti G, Montini L, Pennisi MA, Cavaliere F, Arcangeli A, Bocci $\mathrm{MG}$, et al. A prospective, blinded evaluation of indexes proposed to predict weaning from mechanical ventilation. Intensive Care Med 2004;30(5):830-836.

54. Purro A, Appendini L, De Gaetano A, Gudjonsdottir M, Donner CF, Rossi A. Physiologic determinant of ventilator dependence in longterm mechanically ventilated patients. Am J Respir Crit Care Med 2000;161(4 Pt 1):1115-1123.

55. Jubran A, Grant BJ, Laghi F, Parthasarathy S, Tobin MJ. Weaning prediction: esophageal pressure monitoring complements readiness testing. Am J Respir Crit Care Med 2005;171(11):1252-1259.

56. Mutch WA, Harms S, Ruth Graham M, Kowalski SE, Girling LG, Lefevre GR. Biologically variable or naturally noisy mechanical ventilation recruits atelectatic lung. Am J Respir Crit Care Med 2000;162(1):319-323.

57. Wysocki M, Cracco C, Teixeira A, Mercat A, Diehl JL, Lefort Y, et al. Reduced breathing variability as a predictor of unsuccessful patient separation from mechanical ventilation. Crit Care Med 2006; 34(8):2076-2083

58. Schmidt M, Cecchini J, Kindler F, Similowski T, Demoule A. Breathing variability and mechanical ventilation in intensive care. Reanimation 2014;23:17-24

59. Gutierrez G, Das A, Ballarino G, Beyzaei-Arani A, Türkan H, WulfGutierrez M, et al. Decreased respiratory rate variability during mechanical ventilation is associated with increased mortality. Intensive Care Med 2013;39(8):1359-1367. 
60. Schmidt M, Demoule A, Cracco C, Gharbi A, Fiamma MN, Straus $\mathrm{C}$, et al. Neurally adjusted ventilatory assist increases respiratory variability and complexity in acute respiratory failure. Anesthesiology 2010;112(3):670-681.

61. Sinderby C, Navalesi P, Beck J, Skrobik Y, Comtois N, Friberg S, et al. Neural control of mechanical ventilation in respiratory failure. Nat Med 1999;5(12):1433-1436.

62. Younes M. Proportional assist ventilation, a new approach to ventilatory support: theory. Am Rev Respir Dis 1992;145(1):114-120.

63. Younes M, Puddy A, Roberts D, Light RB, Quesada A, Taylor K, et al. Proportional assist ventilation: results of an initial clinical trial. Am Rev Respir Dis 1992;145(1):121-129.

64. Rose L, Presneill JJ, Johnston L, Cade JF. A randomised, controlled trial of conventional versus automated weaning from mechanical ventilation using smartcare/ps. Intensive Care Med 2008;34(10): 1788-1795.

65. Lee KH, Hui KP, Chan TB, Tan WC, Lim TK. Rapid shallow breathing (frequency-tidal volume ratio) did not predict extubation outcome. Chest 1994;105(2):540-543.

66. Tobin MJ, Chadha TS, Jenouri G, Birch SJ, Gazeroglu HB, Sackner MA. Breathing patterns: 2. diseased subjects. Chest 1983;84(3):286294.

\section{Discussion}

Branson: Of the 11 clinicians here now, how many use automated weaning in their ICU? Let the record show none. The interesting thing to me is I don't perceive this to be something clinicians are wanting to see happen. People aren't sitting in their ICUs today saying, "The reason we can't get people off the ventilators is we don't have automated weaning"; it's way at the bottom of the list. Then you have Dean [Hess] telling us you don't need to wean, ${ }^{1}$ and everybody agrees we shouldn't use intermittant mandatory ventilation (IMV) but that's what ASV (adaptive support ventilation) doesit's SIMV (synchronized intermittent mandatory ventilation) plus pressure support, and it slowly withdraws support. I'm not sure how you can reconcile those 2 things in your mind if you think one is valuable.

Kacmarek: I will agree that none of us use it, and I would not use any of the systems available at the moment. However, I really believe that the future is getting the right algorithm to do automated weaning. We've been weaning by SBTs for many years, and SmartCare automatically initiates an SBT.
67. Bouadma L, Lellouche F, Cabello B, Taillé S, Mancebo J, Dojat M, Brochard L. Computer-driven management of prolonged mechanical ventilation and weaning: a pilot study. Intensive Care Med 2005; 31(10):1446-1450.

68. Yang KL, Tobin MJ. A prospective study of indexes predicting the outcome of trials of weaning from mechanical ventilation. N Engl J Med 1991;324(21):1445-1450.

69. Girard TD, Kress JP, Fuchs BD, Thomason JWW, Schweickert WD, Pun BT, et al. Efficacy and safety of a paired sedation and ventilator weaning protocol for mechanically ventilated patients in intensive care (awakening and breathing controlled trial): a randomised controlled trial. Lancet 2008;371(9607):126-134.

70. Kress JP, Pohlman AS, O'Connor MF, et al. Daily interruption of sedative infusions in critically ill patients undergoing mechanical ventilation. N Engl J Med 2000;342(20):1471-1477.

71. Brochard L, Rauss A, Benito S, Conti G, Mancebo J, Rekik N, et al. Comparison of three methods of gradual withdrawal from ventilatory support during weaning from mechanical ventilation. Am J Respir Crit Care Med 1994;150(4):896-903.

72. Thille AW, Rodriguez P, Cabello B, Lellouche F, Brochard L. Patient-ventilator asynchrony during assisted mechanical ventilation. Intensive Care Med 2006;32(10):1515-1522.
MacIntyre: SmartCare automatically reduces the pressure support and then goes to an SBT.

Kacmarek: But that's not what we do. The point is that what we say we do is not what we actually do. I would bet that in every single one of our ICUs, the perception of how frequently and accurately we do SBTs and reality are grossly different. I will tell you that in our institution, everybody says we do SBTs on a regular basis, but when we go look, no, we don't do SBTs on a regular basis. Patients are missed very commonly. I don't believe that it's better, but I believe it's applied consistently, appropriately, and timely to patients when you have an automated system. But that does not, to John's [Marini] point, imply that it's time to extubate the patient. All it's saying is that the patient can breathe spontaneously independent of the ventilator for a period of time. It still takes the clinician to make the decision to eliminate the artificial airway and to discontinue mechanical ventilation.

MacIntyre: I think we need to clarify our terminology here. The term weaning means a gradual reduction in support: reducing pressure support levels, reducing IMV levels, periodic increases in trach collar or T-piece trials-that's weaning. And I think that needs to be distinguished from an SBT, which is an assessment. They're 2 really different phenomena in our institution. We steer away from this notion that all day long we have to be twirling knobs to reduce pressure support and/or IMV; we don't do that anymore. But what we do mandate is that every morning, usually at the end of the night shift, it's a requirement to do an SBT in people who have certain PEEP, $\mathrm{F}_{\mathrm{IO}_{2}}$, and sedation levels. We do a decent job of that. I think the need to wean in between those assessments is what I like to argue against. I think it wastes clinicians' time, and I think it can actually delay the process of going to an SBT if you have to get to a certain pressure support level before you do one.

Kacmarek: I would bet, Neil, that you don't do as good a job as you think you do.

MacIntyre: I beg to disagree, because we actually monitor it; it's one of our quality assurance measures we monitor. It's one of the few clinical 
things our electronic medical record has figured out how to do.

Kacmarek: Sure, we monitor them too, and the therapists put in, "The patient was assessed for an SBT" every day.

Hurford: To move this along, what you're saying is that these automated techniques ideally adjust the ventilator to the patient's needs. So, to extend that idea, these algorithms should not only be effective in weaning from ventilation, but also adjusting the level of ventilator support throughout the patient's illness. If you really want to extend this, any algorithm that's worth its weight should be an algorithm that can robotically adjust the level of mechanical ventilation throughout the illness, not just at this magic time where the patient may or may not be getting better.

MacIntyre: You're extending the concept to closed-loop ventilation, which is fine, but that's a separate conversation. At least, to me it is. We're talking now about this delay in SBTs until a certain low level of support is there: pressure support of 6, IMV of 2 , or whatever. What I'm arguing is I think that can actually delay things if you wait for your SBTs until you reach a certain low level; I think you run the risk of missing patients who are ready to come off.

Hurford: Would you agree that weaning is a clinical assessment and not just when someone reaches a certain ventilator setting?

MacIntyre: I would not at all. Weaning is a process of gradual reduction in support. An SBT is an assessment, and I think an assessment can be done without weaning.

Hurford: I agree with that.

Mireles-Cabodevila: First, your poll of all of us earlier; we all come from big centers, likely with high staffing models. The application of automatic weaning in smaller centers or long-term acute care facilities with less staffing may be of benefit. Second, about ASV being IMV; yes it is, but when IMV was applied in weaning studies, ${ }^{2}$ it was a human being making the decisions at the bedside. So it has the same problem, regardless of the mode, of us being at the bedside trying to decide when to titrate down the support. ASV uses our input and a set of rules, monitoring the output and regularly applying the rules. Finally, what's interesting about automated weaning is when you use it to standardize research. ${ }^{3}$ This helps to clarify the effects of the intervention on length of mechanical ventilation. You put them on automated weaning, and they will be weaned on the same circumstances. So you can eliminate some of the bias from the decisions made by the teams and application of protocols.

MacIntyre: Assuming the algorithm is a good one.

\section{Mireles-Cabodevila: Yes.}

Kacmarek: It's just a matter of time until we get the correct algorithm. I agree that we don't have it now, but we will, and when we do we'll be much more efficient at using an automated approach than the individual decisions made by me and by you at the bedside because you and I are not at the bedside $100 \%$ of the time.

Marini: I agree that most patients do not need to be gradually weaned. However, it seems that there are patients who need to be accommodated relatively slowly to breathing again naturally. We treat many delirium patients, not just medication delirium but overdoses and such. As a mixed academic/community hospital, we see many patients who are both very difficult to manage and difficult to judge whether they're ready for extubation.
If we were to rely only on protocols, only on automated weaning, or only on sporadic physician observations, we're likely to catch the patient at the wrong time, and it's not likely to work. We need to integrate visual cues and trends into the decision. Here, as usual, I agree with Bob-we do need to consider automated weaning as something that's going to happen in the future. We're going to have fewer people available. But there's an irreducible level of chaos in the clinical setting that can derail our best intentions or the program of a machine. An ideal automated system will remind us when we're going wrong and maybe call it to our attention.

Holets: Exactly. What we see with a patient recovering from ARDS is we try to go from a fully supportive mode to you're getting liberated from the vent. What happens is they start double triggering, they start flowstripping, nobody's around, the nurse re-sedates the patient, and we miss that opportunity to maybe give them partial support and get them weaned that day.

Kallet: I want to trademark this, but my major problem is that closed-loop ventilation closes the clinicians out of the loop. And if I may create a new word, I think a lot of this "stupidifies" clinicians. As I see more of this happen, particularly with things like autoflow, I see clinicians lose their ability to assess a patient. Every time we're called into the room to make an adjustment, as a clinician you're looking at the patient. When you do something automated, at the point where we reach neural nets and can integrate a lot of the data, I think we'll be there. But after 40 years of doing this, I'm not convinced that automating things is going to make it better. Point of fact (from our SBT database): Of patients with ARDS who have been ventilated for $10 \mathrm{~d}$ before they met SBT criteria, $50 \%$ of them pass their first SBT for $2 \mathrm{~h}$ without a problem. It's not that 
big of a deal. I think it's not weaning, and they have a whole industry geared toward a small percentage of the population, and I think they're barking up the wrong tree.

Hess: I'll follow up on that a little bit. John mentioned the problem with the sensors, and I agree with that. I think one of the problems that comes with these approaches is we get garbage in/garbage out. Certainly, setting the ventilator based on end-tidal $\mathrm{P}_{\mathrm{CO}_{2}}$ is, I think, very problematic from a physiologic standpoint. As far as improving the sensors so that this can be done better, there is one thing that I think is important, which is the eyeball test. I don't know how we come up with a sensor that replaces the eyeball test, where you are at the bedside and you see the diaphoresis, the eyes wide open, and the neck muscle contraction. Those things are going to be difficult to automate, and it drives a lot of our clinical decision making.

Marini: I don't know of any experienced physician who does not go into the room of a patient and without looking at anything else senses that the patient has turned the corner and looks better (or the opposite) - the eyeball test! It's exactly as you're saying. We unconsciously take in and integrate so much information. For example, when we come back to this room after lunch, I will recognize almost everything in here because our brains automatically put it in all in perspective. Your brain is continually processing all this information, even if you don't intentionally commit it to memory. You recognize everything, and every face you know. You were not aware of memorizing, but unconsciously, you have placed it in memory because your brain has taken it all in and figures it out. There's a lot we don't understand about perception and the integration of information that human observation adds.
Holets: A comment going back to Rich, where this will "stupidify" usI'll quote my mentor, Dr Hubmayr, who says pressure support is the most widely used and misunderstood mode out there. It's easy to recognize weaning failure when we see the patient whose eyes are bugging out, but how many difficult-to-wean patients have you seen who have been on low levels of pressure support and PEEP for 5 days, and no one recognizes they are totally exhausted? All they're doing is triggering the ventilator, and nobody recognizes it as weaning failure.

Kacmarek: I've been doing this for 50 years and I' $m$ convinced that the only way we're going to get it right is if we automate it. Because we don't have the people and we don't have the skilled people at the bedside every minute. There was an abstract ${ }^{4}$ at ATS this year that talked about asynchrony, and they took videos of patients during asynchrony, and they were able to identify facial changes and body movements that were associated with high levels of asynchrony. There's no reason we can't develop automated systems that also video the patient simultaneously and can interpret those kind of issues you're making reference to. But I don't think anybody is proposing that an automated system at any level will eliminate clinicians. I don't think it would eliminate the RT, physician, or nurse. It takes all of them to work with the system to properly apply it and to ensure that it's done properly. What it does ensure is that it's always done the same way and that the interpretation is appropriate and continuous; we don't do that today. You are lying to yourselves if you think we are doing that in the ICU today because we are not. There are huge inconsistencies, depending on the RTs, the nurses, and the physicians who are in the unit as well as the issues ongoing at any one point in time in the unit.
Branson: I agree that it improves consistency. Weaning or closed-loop ventilation provides consistency and gets rid of some of the variability that we see because of which RT is on or what doc is on. But whether it will be better I think remains to be seen.

Mireles-Cabodevila: As long as it's not worse!

Kacmarek: Note that not a single study $^{5-9}$ showed it was worse. They showed it was equivalent, but not a single study showed that it was worse than clinician-guided weaning or SBTs.

Hess: But how much does it cost? What is the cost-effectiveness of this? They don't make ventilators cheaper to have this.

Marini: It might add $\$ 5,000$ to $\$ 7,000$ (or something like that) to have that feature.

Holets: It depends on how many vents you buy; the more vents, the less the option costs.

MacIntyre: I'd rather spend it on therapists.

\section{REFERENCES}

1. Hess DR, MacIntyre NR. Ventilator discontinuation: why are we still weaning? Am J Respir Crit Care Med 2011;184(4): 392-394.

2. Esteban A, Frutos F, Tobin MJ, Alía I, Solsona JF, Valverdú I, et al. A comparison of four methods of weaning patients from mechanical ventilation: Spanish Lung Failure Collaborative Group. N Engl J Med 1995; 332(6):345-350.

3. Mekontso Dessap A, Roche-Campo F, Kouatchet A, Tomicic V, Beduneau G, Sonneville R, et al. Natriuretic peptidedriven fluid management during ventilator weaning: a randomized controlled trial. Am J Respir Crit Care Med 2012;186(12): 1256-1263.

4. Mellott KG, Grap MJ, Munro CL, Sessler CN, Wetzel PA, Nilsestuen JO, Ketchum JM. Patient ventilator asynchrony: associated patient behaviors. Am J Respir Crit Care Med 2015;191:A3897. 
5. Lellouche F, Mancebo J, Jolliet P, Roeseler J, Schortgen F, Dojat M, et al. A multicenter randomized trial of computer-driven protocolized weaning from mechanical ventilation. Am J Respir Crit Care Med 2006; 174(8):894-900.

6. Kataoka G, Murai N, Kodera K, Sasaki A, Asano R, Ikeda M, et al. Clinical experience with Smart Care after off-pump cor- onary artery bypass for early extubation. J Artif Organs 2007;10(4):218-222.

7. Rose L, Presneill JJ, Johnston L, Cade JF. A randomised, controlled trial of conventional versus automated weaning from mechanical ventilation using SmartCare/PS. Intensive Care Med 2008;34(10):1788-1795.

8. Schädler D, Engel C, Elke G, Pulletz S, Haake N, Frerichs I, et al. Automatic con- trol of pressure support for ventilator weaning in surgical intensive care patients. Am J Respir Crit Care Med 2012;185(6):637-644.

9. Burns KE, Meade MO, Lessard MR, Hand L, Zhou Q, Keenan SP, Lellouche F. Wean earlier and automatically with new technology (the WEAN study): a multicenter, pilot randomized controlled trial. Am J Respir Crit Care Med 2013;187(11):1203-1211.

This article is approved for Continuing Respiratory Care Education credit. For information and to obtain your CRCE

(free to AARC members) visit

www.rcjournal.com 